\title{
GDPs' attitudes to clinical governance
}

\author{
Attitudes and opinions of NHS general dental practitioners towards clinical governance
}

R. J. McCormick and J. W. Langford Br Dent J 2006; 200: 214-217

\section{Aim}

To assess the attitudes and opinions of NHS general dental practitioners towards clinical governance.

Design

This was a questionnaire based study, sent to NHS principal dentists within the West Midlands area.

Method

A Likert scale questionnaire was developed, consisting of 26 statements in 4 subject areas. It was internally and externally validated, and sent to 208 practices within four geographic areas.

Results

A total of 150 questionnaires were returned; a response rate of $72 \%$. For each question, no significant difference was found between areas. The cost and time involved with clinical governance emerged as the most important issues, with many respondents considering that costs of implementation might make more dentists leave the NHS. Dentists were largely positive about the principles of clinical governance and evidence based practice, but were concerned about the possibility of increasing complaints and some doubted that it would result in improved patient care. Many respondents claimed to be still confused about clinical governance and the majority considered that more guidance should be available to assist with development within dental practice.

Conclusions

This survey showed that some problems exist around the introduction of clinical governance within NHS general dental practice.

\section{IN BRIEF}

- GDPs perceive clinical governance to be good for dentistry but its introduction is likely to encounter some resistance.

- Lack of guidance, time and cost of implementation are seen as the main barriers to the introduction of clinical governance within dentistry.

- Most dentists consider risk management important and clinical governance is making them more aware of risk.

- Dentists' issues of concern are very similar to those of medical practitioners when clinical governance was introduced into medical practice.

\section{COMMENT}

As stated at the beginning of this paper, clinical leadership and organisational culture must change if the qualities of care are to improve. Since the formalisation of clinical governance, in particular its incorporation into the Terms and Conditions of Service, there have been several positive improvements in aspects of the care system. These improvements include the greater acceptance of evidence informed health care and a willingness to challenge current practices. However, as one of the findings of this paper highlights, the improvements are fragmented.

With nearly two thirds of respondents undecided or agreeing that they are confused about clinical governance there is a problem. If the care delivery system is to improve then clarity of meaning is of paramount importance. Words such as 'high quality' and 'equity' commonly found in many Departmental publications lack precision. If dentists are to embrace the laudable principles of clinical governance this shortfall must be addressed.

All health care delivery arrangements are formed of highly complex systems. Complexity creates a barrier to change: it creates uncertainties and fear. To overcome fear and uncertainty in complex systems requires management to specify the direction in which the arrangements should move, specifying the minimum requirements and allowing autonomy to achieve the change. If those tasked with changing do not understand how they can modify their practices to meet the requirements of any corporate strategy, a reluctance to alter will arise. However, knowledge alone does not necessarily bring out change. As Mason et al. ${ }^{1}$ highlight, multifaceted strategies are required.

Perhaps the most interesting finding lies within the section on clinical governance and dental policy. At the time of the study there was considerable uncertainty about the future direction of the NHS care delivery system. However, clinical governance is about the care system in total. Whether care is delivered through NHS or other arrangements should not matter: improving the qualities of care should be central to any organisation. The growing cynicism of the profession about the future will not help. The authors of the present paper open their conclusion by stating that the biggest change in the provision of NHS dentistry is about to be implemented, giving rise to the important challenge facing Primary Care Trusts (PCTs) with the need to become aware of the problems. The additional upheaval of the reform programme with a decline in the number of PCTs at the same time can hardly be conducive to a positive outcome. But equally, the uncertainty for PCTs does provide practitioners with the opportunity to show leadership and take ownership of what should be a basic requirement of care provision.

P. Batchelor, Honorary Consultant in Dental Public Health, Eastman Dental Institute

1. Mason A, Ingham J, Parnham J. Making clinical governance work. Clin Med 2005: 5: $122-125$

doi: 10.1038/sj.bdj.4813256 